\title{
Ultrasound Medical Image Denoising using Hybrid Bilateral Filtering
}

\author{
V Naga Prudhvi Raj \\ Assoc.Professor, EIE Department, \\ VR Siddhartha Engg. College \\ Vijayawada, India
}

\author{
T Venkateswarlu, PhD. \\ Professor, ECE Department \\ SV University College of Engineering \\ SV University, Tirupati, India.
}

\begin{abstract}
Medical imaging is placing a major role in diagnosing the diseases and in image guided surgery. There are various imaging modalities for different applications giving the anatomical and physiological conditions of the patient. All these modalities will introduce some amount of noise and artifacts during medical image acquisition. If the noise and artifacts are not minimised diagnosis will become difficult. One of the non-invasive modality is ultrasound where no question of radiation but suffers from speckle noise produced by the small particles in the tissues who's size is less than the wavelength of the ultrasound. The presence of the speckle noise will cause the low contrast images where low contrast lesions and tumors can't be detected in the diagnostic phase. So there is a strong need in developing the despeckling techniques to improve the quality of ultrasound images.Many image denoising techniques based on spatial filtering, total variational filtering, bilateral filtering and multiresolution filtering etc. In most of the filtering techniques the objective is removing the noise while preserving the edges in the image. Still research is going on in the improvement of denoising procedures without losing the diagnostic details. Here in this paper we are proposing a method which will combine the bilateral filtering and multiresolution approach (Discrete Wavelet Transform) to remove the noise from ultrasound medical images. The advantage of this method is it removes the noise in the approximation subband or low frequency subband in the wavelet decomposition. The performance of the filtering was evaluated using several image quality metrics and the results showed that the proposed hybrid filter is outperforming the methods based on wavelet transforms and spatial filtering.
\end{abstract}

\section{General Terms}

Multifocus Image Fusion, Multi Resolution Analysis

Keywords

Image Fusion, Discrete Wavelet Transform, Image Gradient.

\section{INTRODUCTION}

Medical imaging developments in the last few decades becoming helpful in diagnosis by observing the anatomical structures without opening the human body and treatment of complicated diseases such as carotid artery stenosis where a plaque is going to build up in the arteries causing the brain stroke through blocking of blood flow from heart to brain, brain tumor localisation and size of the tumor, early detection of arthritis etc. There are many imaging modalities developed such as X-ray's, Computed Tomography, Ultrasound, Nuclear medicine (Positron Emitted Tomography (PET), Single positron Emitted Computed Tomography SPECT), and
Magnetic Resonance imaging etc. for various applications. Hybrid modalities such as PET/CT, PET/MRI etc are useful to study the dynamical behaviour of human tissues along with their anatomical structures [19].

These medical images are different from natural photographic images where the images are acquired by capturing the reflected light from the object. Here in medical imaging processes images are acquired for clinical procedures reflect complex physical and physiological phenomena of many different types. While forming the images every modality introduce certain amount of noise and artifacts which will complicate the diagnostic process by blurring the details and hiding the tumors etc. So the need for denoising is very important step before submitting the medical images for diagnostic phase. In this paper we are concentrating on presence of noise in ultrasound medical images and its denoising procedures developed using spatial filtering and multiscale transforms such as discrete wavelet transform [21], Undecimated wavelet transform [25], Dual tree complex wavelet transforms [29] and Double density dual tree complex wavelet transforms [32].

The noise may be additive or multiplicative depending on the modality used for medical image acquisition. The noise due to electronic components in the acquisition hardware will be modeled with Gaussian noise which independent of data, the data dependent noise such as quantum noise in X-ray imaging is modeled with poisson distribution, the speckle noise in ultrasound imaging is modeled with Rayleigh distribution and the noise in MRI is modeled with Rician distribution. Here in this paper we are attempting to denoise the images corrupted with speckle noise.

The speckle noise reduces the contrast resolution of the acquired image by ultrasound imaging modality. Because of this the detection of low contrast lesions and tumors will become difficult in diagnostic phase. The destructive interference of sound waves scattered from various sites will produce the speckle pattern in the images [1]. The speckle pattern in the image visible as light and dark spots. Under the same conditions if a fixed object is scanned twice the speckle pattern produced in both the scans is identical. However if the scanning conditions such as different transducer aperture, transducer angulation or pulse length were changed the speckle pattern will also change [2]. Speckle noise is produced by scattering phenomena. Scattering is a process where sound waves are forced to deviate from its path by one or more localised non-uniformities in the tissue through which they pass. These non-uniformities are called as scatterers or scattering centers. In a tissue the scattering centers arises due to inhomogeneity or small structures whose size is less than the wavelength of the ultrasound [1]. Tissue parenchyma is an 
example where there is a change in acoustic impedance over a microscopic level within the tissue.

The scattering or speckling is caused by the tissue particles which are smaller than the wavelength of the ultrasound such as blood cells and particles that are having different acoustic impedance which are close to one another.The mathematical modeling of degradation and restoration process is given as

$$
\begin{aligned}
& g(x, y)=f(x, y) * h(x, y)+\eta(x, y) \\
& G(u, v)=F(u, v) H(u, v)+N(u, v)
\end{aligned}
$$

Where $g(x, y)$ is the noisy and blurred observation, $H$ is the blurring kernel and $f(x, y)$ is the signal we are recovering. In the case of denoising problem the blurring kernel will be dropped and the degradation model will be given as

$$
\begin{aligned}
& g(x, y)=f(x, y)+\eta(x, y) \\
& G(u, v)=F(u, v)+N(u, v)
\end{aligned}
$$

In the case of multiplicative noise the model is given as

$$
g(x, y)=f(x, y) \cdot \eta(x, y)
$$

Since the evolution of digital imaging many techniques were developed to remove the noise from the noisy images. Earlier the techniques were based on point processing i.e modifying the pixel intensity using some linear transformation which is not adaptive to the noise content in the image and all the pixels were processed by the same filtering rule. Due to this the filter cannot differentiate the smooth areas and edges in the image and will smooth the entire image. Because of this the edges are going to be blurred or will lose contrast so the resulting image is not an optimal solution for performing diagnostic examinations [5].

Later the neighborhood processing became popular by considering the neighboring pixel intensities while modifying the pixels intensity. In this paper we are trying to adapt the principles of these filters for the removal of speckle from the ultrasound medical images. One of the major directions in image denoising is modeling the image using partial differential equations and adopting the principles of diffusion from thermodynamics into the image denoising procedure. The famous algorithms in this category is diffusion filtering, anisotropic diffusion filtering etc [6-10].

Another major direction which we are using in this paper is multiscale transforms. These transforms decomposes the noisy images into various scales and subbands and each scale and subband coefficients are processed to remove the noise from these subbands and finally the approximated image to the original image is reconstructed. The major advantage of these transforms is energy compaction and localisation of singularities such as points, lines and edges etc.

In our paper the second section will discuss the implementation of six spatial filters for the speckle noise removal. In the third section discrete wavelet transform (DWT), Undecimated Wavelet Transform (UDWT), section fourth section dual tree complex wavelet transform (DTCDWT) and double density dual tree complex wavelet transform are discussed. The fifth section will present the various Image quality assessment metrics are presented. The sixth section will present the performance of various filters in denoising the speckle noise from ultrasound medical imaging and the seventh section concludes the paper.

\section{BILATERAL FILTERING}

In spatial filtering of images the filtering process is carried out by running the neighborhood masks (filter, template, and kernel) on the image from left to right and top to bottom. The center coefficient of the filter is placed on the pixel to be modified and all the four neighbors or eight neighbors are taken into consideration while performing the filtering operation. The best example is performing the Gaussian filtering on the image whose weights are decreasing with the distance from the center pixel. The Gaussian filtering will remove the noise but fails to preserve the edges. Many techniques were introduced in the literature to denoise the images while preserving the edge features. Here we are proposing the bilateral filtering with the multiresolution transform.

The meaning of bilateral is affecting or undertaken by two sides equally. Here in the bilateral filtering the main idea is perform the filtering both in the domain and the range of the image. That is smooth as usual in the domain of the image and do not smooth when pixels are not similar (Possibility of edge). The filter performs strong smoothing when similar pixels are present otherwise no smoothing is performed. This similarity among pixels are identified by the similarity function

Based on intensity values of pixels two pixels are considered similar if they have the same value. The filtering process is explained with the following mathematical equations. Let $S$ is the central pixel in the neighborhood $\Omega$ of size $5 \times 5$ or $7 \times 7$ whose value is going to be modified by the filter and $p$ be the . $I_{p}$ and $I_{s}$ are the intensity values of $p$ and $s$

$$
\begin{aligned}
& J_{s}=\frac{1}{k_{s}} \sum_{p \in \Omega} f(p-s) g\left(I_{p}-I_{s}\right) I_{p} \\
& k_{s}=\sum_{p \in \Omega} f(p-s) g\left(I_{p}-I_{s}\right)
\end{aligned}
$$

$f(p-s)$ is the closeness function measures geometric distance between $p_{\text {and }} s . g(p-s)$ is the photometric similarity function measures photometric similarity between

$I^{p}$ and ${ }^{I_{s}}$. Combined domain and range filtering will be denoted as bilateral filtering. It replaces the pixel value at $p$ with an average of similar and nearby pixel values. In smooth regions, pixel values in a small neighborhood are similar to each other, and the bilateral filter acts essentially as a standard domain filter, averaging away the small, weakly correlated differences between pixel values caused by noise. The closeness and similarity functions are given below

\section{Domain weighting}

$$
\begin{aligned}
& f(p-s)=e^{-\frac{d(p-s)^{2}}{2 \sigma_{d}^{2}}} \\
& d(p-s)=\|p-s\|=\sqrt{p^{2}+s^{2}}
\end{aligned}
$$


Range Weighting

$$
\begin{aligned}
& g\left(I_{p}-I_{s}\right)=e^{-\frac{\delta\left(I_{p}-I_{s}\right)^{2}}{2 \sigma_{r}^{2}}} \\
& \delta\left(I_{p}-I_{s}\right)=\left\|I_{p}-I_{s}\right\|
\end{aligned}
$$

\section{DENOISING USING DWT}

In this section we want to use the multiscale transforms for the image denoising because they are very much useful to isolate the discontinuities present in the image and to handle the nonstationary signals or time varying signals. The spatial domain filtering discussed above is succeeded to some extent by introducing the adaptivity in the filtering scheme through first order and higher order statistics at the cost of computational cost and leaving few artifacts such as ringings and smoothing the edges. In some filters the computational cost is too high so that they are not optimal for real time filtering. To overcome these limitations lot of research was taken place in the last two decades.

The multiscale transforms such as gaussian and laplacian pyramids,steerable pyramids and wavelets are performing well in many image processing tasks by decomposing the images into multiple scales and using the benefit of sparsity and energy compaction of the above transforms that is representing the most of the information in very few coefficients. In this paper we are denoising the images using wavelet transform, undecimated wavelet transform, dual tree complex wavelet transform and double density dual tree complex wavelet transform and compared the denoising performance with various quality metrics along with observing the effect of denoising on texture of the medical images which is a very important factor while choosing the denoising algorithm.

\subsection{Discrete Wavelet Transform}

The DWT of a signal $\mathrm{x}(\mathrm{n})$ is calculated by passing it through a series of filters. First the samples are passed through a low pass filter with impulse response resulting in a convolution of the two [21]:

$$
y[n]=\left(x * h_{0}\right)[n]=\sum_{k=-\infty}^{\infty} x(k) h_{0}(n-k)
$$

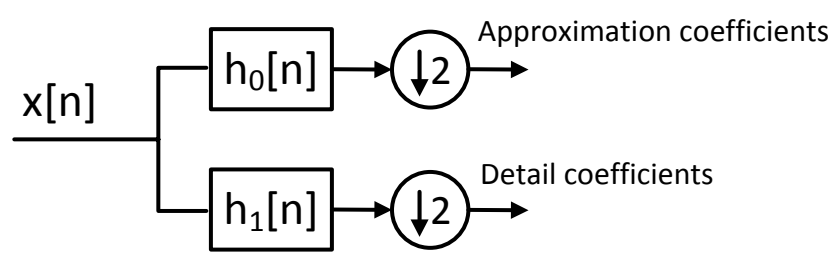

Figure 1: 1D Analysis filters

The signal is also decomposed simultaneously using a highpass filter. The outputs of the lowpass filter are approximation coefficients and highpass filter are detail coefficients. It is important that the two filters are related to each other and they are known as a quadrature mirror filter. Since half the frequencies of the signal have now been removed, half the samples can be discarded according to Nyquist's rule. The filter outputs are then subsampled by 2 .

$$
\begin{aligned}
& y_{\text {low }}[n]=\sum_{k=-\infty}^{\infty} x(k) h_{0}(2 n-k) \\
& y_{\text {high }}[n]=\sum_{k=-\infty}^{\infty} x(k) h_{1}(2 n+1-k)
\end{aligned}
$$

2D DWT of the images can be implemented by applying 1D DWT along the rows of an image first and then applying 1D DWT on the columns of an image. When a wavelet transform is applied to an image the image is decomposed into four subbands as shown in the following figure 4 . The LL band contains the approximation coefficients, LH band contains horizontal details, HL band contains vertical details and $\mathrm{HH}$ band will contain the diagonal details [21].

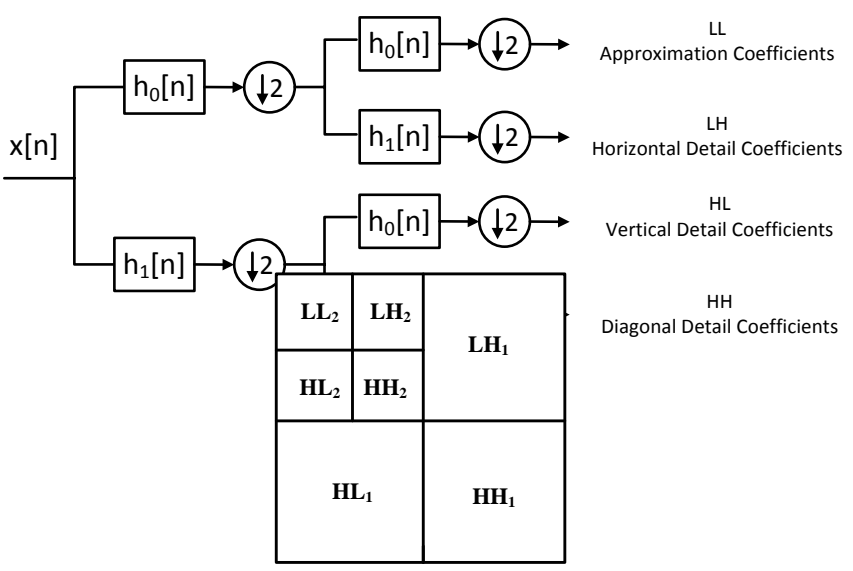

Figure 2: 2D Analysis filters and Image decomposition

\subsection{Denoising Procedure using Multiscale Transforms}

1. Compute the forward transform of the image to be denoised and decompose the image into subbands

2. Compute the threshold from the first scale $\mathrm{HH}$ (vertical details) band using the MAD (median absolute deviation) using the following formula considering that most of the noise is present in that band.

$$
\hat{\sigma}(\text { mad })=\frac{\text { median }\left\{\left|w_{j}\right|: j=1,2, \ldots \frac{k}{2}\right\}}{0.6745}
$$

3. Apply the shrinkage step (modifying the wavelet coefficients in the subbands) using the following shrinkage rules. [22, 23, 24]

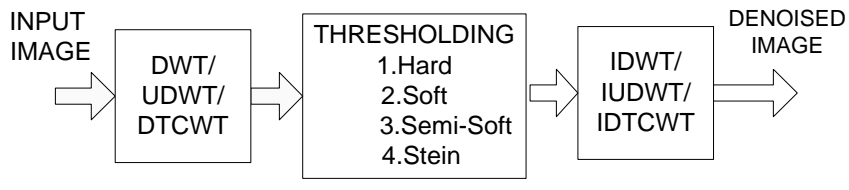

Figure 3: Image denoising using Multiscale Transforms 


\section{Shrinkage Rules}

\section{Hard Thresholding}

$$
D_{H}^{T}(w)=\left\{\begin{array}{cc}
w & \text { for all }|w|>T \\
0 & \text { otherwise }
\end{array}\right.
$$

Soft Thresholding

$$
D_{H}^{s}(w)=\operatorname{sgn}(w) \max (0 ;|w|-T)
$$

\section{Semi-soft Thresholding}

$$
D_{s s}^{T T 1}(w)=\left\{\begin{array}{cc}
0 & |w| \leq T \\
\operatorname{sgn}(w) \frac{T 1(|w|-T)}{T 1-T} & T<|w| \leq T 1 \\
w & |w|>T 1
\end{array}\right.
$$

4. After modifying the wavelet coefficients in the subbands take the inverse transform to reconstruct the image to get denoised image which is an estimation of the original one.Many shrinkage rules are associated with the wavelet processing. The threshold may be calculated globally, level dependent or subband dependent. But here we are calculating the threshold globally.

\section{PROPOSEDMETHOD}

Multiresolution analysis is the important tool in noise elimination from signals and images effectively. In MRA analysis it is possible to distinguish the noise and image information better at one resolution level than another. Here we are proposing the method which combines the features of bilateral filtering and multi resolution analysis to get the benefit from both the methods. The proposed framework is shown in the following figure metrics are classified as pixel difference based measures, correlation based measures, edge based measures, spectral distance measures, context based measures and Human visual system based measures. Here we are comparing our denoising algorithms using a group of metrics drawn from the above class and performance of the algorithms was observed.

\subsection{Pixel difference based measures}

\subsubsection{Minkowski metrics}

The norm of the dissimilarity of two images can be calculated by calculating the minkowski average of the pixel differences spatially and then chromatically as given below

$$
\varepsilon^{\gamma}=\frac{1}{K} \sum_{k=1}^{K}\left\{\frac{1}{M N} \sum_{x=0}^{M-1} \sum_{x, y=0}^{N-1}\left|f_{k}(x, y)-\hat{f}_{k}(x, y)\right|^{\gamma}\right\}^{\frac{1}{\gamma}}
$$

Where $f(x, y)$ is the reference image, $\hat{f}(x, y)$ is the estimated image of $f(x, y)$ by our denoising algorithm with the input $g(x, y)$ which is a noisy version of $f(x, y)$.

For $\gamma=1$ we obtain the absolute difference (AD), for $\gamma=2$ we will obtain the mean square error (MSE). Along with these two measures we are calculating minkowski measures for $\gamma=3$ and $\gamma=4$ in this paper to observe the performance of our algorithms.

\subsubsection{PSNR (Peak Signal to Noise Ratio)}

PSNR is the peak signal-to-noise ratio in decibels $(\mathrm{dB})$. The PSNR is only meaningful for data encoded in terms of bits per sample, or bits per pixel. For example, an image with 8 bits per pixel contains integers from 0 to 255 .

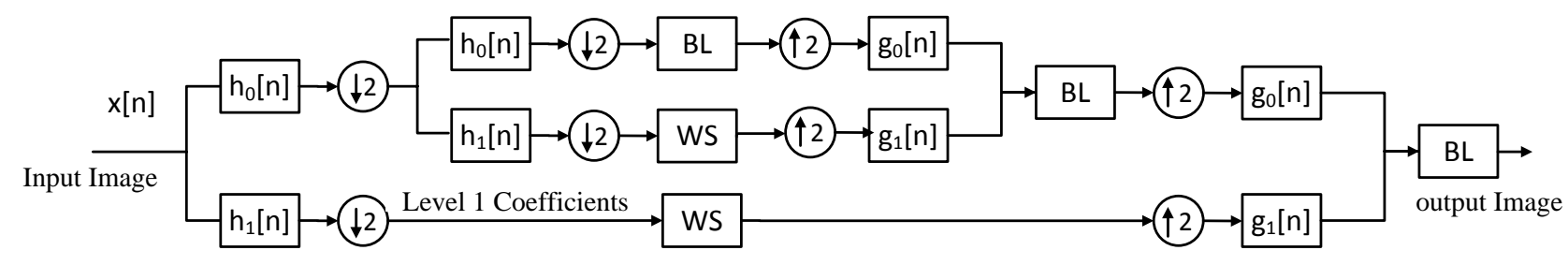

Here in the proposed filtering the image is decomposed into various levels and subbands using discrete wavelet transform. Then the high frequency details coefficients are modified using wavelet shrinkage using any one of the shrinkage rule. The low frequency subband is modified using the bilateral filtering. The advantage of this filtering process is the noise present in the low frequencies are also removed along with the noise present in the high frequency subbands with edge preserving feature. In the reconstruction side after every stage we filter the signal with the bilateral filter to improve the edge enhancing capability.

\section{PERFORMANCE EVALUATION}

To evaluate the quality of the image processing algorithms there are several metrics proposed in the literature. The

$$
P S N R=20 \log _{10}\left(\frac{2^{B}-1}{\sqrt{M S E}}\right)
$$

Where B represents bits per sample and MSE (Mean Squared error) is the mean square error between a signal $f(x, y)$ andan approximation $\hat{f}(x, y)$ is the squared norm of the difference divided by the number of elements in the signal.

$$
\begin{aligned}
M S E & =\|f(x, y)-\hat{f}(x, y)\|^{2} \\
& =\frac{1}{M N} \sum_{x=0}^{M} \sum_{y=0}^{N}[f(x, y)-\hat{f}(x, y)]^{2}
\end{aligned}
$$




$$
R M S E=\sqrt{\frac{1}{M N} \sum_{x=0}^{M} \sum_{y=0}^{N}[f(x, y)-\hat{f}(x, y)]^{2}}
$$

\subsubsection{Maximum Difference}

Maximum difference is defined as

$$
M D=\max (|f(x, y)-\hat{f}(x, y)|)
$$

The large value of maximum difference means denoised image is poor quality.

\subsubsection{Normalised Absolute Error (NAE)}

The large value of normalized absolute error means that denoised image is poor quality and is defined as

$$
N A E=\frac{\sum_{x=0}^{M-1} \sum_{y=0}^{N-1}|f(x, y)-\hat{f}(x, y)|}{\sum_{x=0}^{M-1} \sum_{y=0}^{N-1}|f(x, y)|}
$$

\subsubsection{Signal to Noise Ratio (SNR)}

Signal to noise ratio in an image is calculated as

$$
S N R=\frac{\mu}{\sigma}
$$

Where $\mu$ is the average information in the signal and $\sigma$ is the standard deviation of the signal which represents the amount of noise present in the image. There is one more measure is there similar to the SNR it is signal to background ratio.

$$
S B R=\frac{\mu}{\sigma_{B G}}
$$

Subtract background from the image calculate standard deviation from it and finally compute the above ratio.

\subsection{Correlation based measures}

The correlation between two images can also be quantified interms of correlation function. These measures measure the similarity between the two images hence in this sense they are complementary to the difference based measures.

\subsubsection{Structural content}

For an $M \times N$ image the structural content is defined as

$$
S C=\frac{1}{K} \sum_{k=1}^{K} \frac{\sum_{x=0}^{M-1} \sum_{y=0}^{N-1} f_{k}(x, y)^{2}}{\sum_{x=0}^{N-1} \sum_{y=0}^{N-1} \hat{f}_{k}(x, y)^{2}}
$$

The large value of structural similarity means that denoised image is poor quality

\subsubsection{Normalised cross correlation measure (NK)}

The normalized cross correlation measure is defined as

$$
N K=\frac{1}{K} \sum_{k=1}^{K} \frac{\sum_{x=0}^{M-1} \sum_{y=0}^{N-1} f_{k}(x, y) \hat{f}_{k}(x, y)}{\sum_{x=0}^{M-1} \sum_{y=0}^{N-1} f_{k}(x, y)^{2}}
$$

\subsubsection{Czekanowski distance}

A metric useful to compare vectors with strictly positive components as in the case of images is given as

$$
C=\frac{1}{M N} \sum_{x=0}^{M-1} \sum_{y=0}^{N-1}\left[1-\frac{2 \sum_{k=1}^{K} \min \left(f_{k}(x, y), \hat{f}_{k}(x, y)\right)}{\sum_{k=1}^{K} f_{k}(x, y)+\hat{f}_{k}(x, y)}\right] \text { (23) }
$$

This coefficient is also called as percentage similarity measures the similarity between different samples, communities and quadrates.

\subsection{Edge Based metrics}

\subsubsection{Laplacian Mean Square Error (LMSE)}

This measure is based on importance of edges measurement. The large value of Laplacian mean square error means that the image is poor quality. LMSE is defined as

$$
L M S E=\frac{\sum_{x=0}^{M-1} \sum_{y=0}^{N-1}[L(f(x, y))-L(\hat{f}(x, y))]^{2}}{\sum_{x=0}^{M-1} \sum_{y=0}^{N-1}[L(f(x, y))]^{2}}
$$

\subsection{HVS based metrics}

\subsubsection{Universal Image Quality Index (UQI)}

It is a measure used to find the image distortion. It is mathematically defined by making the image distortion relative to the reference image as a combination of three factors: Loss of correlation, Luminance distortion and contrast distortion.

If two images $f(x, y)$ and $\hat{f}(x, y)$ are considered as a matrices with $\mathrm{M}$ column and $\mathrm{N}$ rows containing pixel values $f(x, y)$ and $\hat{f}(x, y)$ respectively the universal image quality index $\mathrm{Q}$ may be calculated as a product of three components

$$
Q=\frac{\sigma_{\hat{f f}}}{\sigma_{f} \sigma_{\hat{f}}} \cdot \frac{2 \bar{f} \overline{\hat{f}}}{f^{2}+\hat{f}^{2}} \cdot \frac{2 \sigma_{f} \sigma_{\hat{f}}}{\sigma_{f}^{2}+\sigma_{\hat{f}}^{2}}
$$

Where $\quad \bar{f}=\frac{1}{M N} \sum_{x=0}^{M-1} \sum_{y=0}^{N-1} f(x, y)$ and

$\overline{\hat{f}}=\frac{1}{M N} \sum_{x=0}^{M-1} \sum_{y=0}^{N-1} \hat{f}(x, y)$ 
$\sigma_{\hat{f f}}=\frac{1}{M+N-1} \sum_{x=0}^{M-1} \sum_{y=0}^{N-1}(f(x, y)-\bar{f})(\hat{f}(x, y)-\overline{\hat{f}})$

$\sigma_{f}^{2}=\frac{1}{M+N-1} \sum_{x=0}^{M-1} \sum_{y=0}^{N-1}(f(x, y)-\bar{f})^{2}$ and

$\sigma_{\hat{f}}^{2}=\frac{1}{M+N-1} \sum_{x=0}^{M-1} \sum_{y=0}^{N-1}(\hat{f}(x, y)-\overline{\hat{f}})^{2}$

The first component is the correlation coefficient which measures the degree of linear correlation between images. It varies in the range $[-1,1]$. The best value 1 is obtained when the images are linearly related. The second component measures how close the mean luminance is between images with a range $[0,1]$. The third component measures the contrasts of the images the value range for this component is $[0,1]$. The range of values for $\mathrm{Q}$ is $[-1,1]$. The best value 1 is achieved if and only if the images are identical.

5.4.2 Structural similarity (SSIM) index is a method for measuring the similarity between two images [28]. The SSIM index is a full reference metric, in other words, the measuring of image quality based on an initial uncompressed or distortion-free image as reference. SSIM is designed to improve on traditional methods like peak signal-to-noise ratio (PSNR) and mean squared error (MSE), which have proved to be inconsistent with human eye perception [10].

The SSIM metric is calculated on various windows of an image. The measure between two windows $x$ and $y$ of common size $N \times N$ is [28]:

$$
\operatorname{SSIM}(x, y)=\frac{\left(2 \mu_{x} \mu_{y}+c_{1}\right)\left(2 \sigma_{x y}+c_{2}\right)}{\left(\mu_{x}^{2}+\mu_{y}^{2}+c_{1}\right)\left(\sigma_{x}^{2}+\sigma_{y}^{2}+c_{2}\right)}
$$

Where

$>\mu_{x}$ is the average of $x$ and $\mu_{y}$ is the average of $y$

$>\sigma_{x}^{2}$ is the variance of $x$ and $\sigma_{y}^{2}$ is the variance of $y$

$>\sigma_{x y}$ is the covariance of $x$ and $y$

$>c_{1}=\left(k_{1} L\right)^{2}, c_{2}=\left(k_{2} L\right)^{2}$ two variables to stabilize the division with weak denominator

$>\quad L$ is the dynamic range of the pixel values (typically this is $2^{\# \text { bits per pixel }}-1$ )

$>\quad k_{1}=0.01$ and $k_{2}=0.03$ by default

In order to evaluate the image quality this formula is applied only on luma. The resultant SSIM index is a decimal value between -1 and 1 , and value 1 is only reachable in the case of two identical sets of data. Typically it is calculated on window sizes of $8 \times 8$. The window can be displaced pixel-by-pixel on the image.

\section{RESULTS\&CONCLUSIONS}

The ultrasound image corrupted with speckle noise is given as input to the bilateral filter, DWT filter, and the proposed filter and the above mentioned image quality metrics were computed. Based on the values obtained and the visual perception the proposed method is performing well in despeckling the ultrasound medical images.

Table 1: Performance of bilateral and DWT filters

\begin{tabular}{|c|c|c|c|c|}
\hline & $\begin{array}{l}\text { Bilateral } \\
\text { Filtering }\end{array}$ & \multicolumn{3}{|c|}{ DWT Filtering } \\
\hline & & Soft & Hard & Semisoft \\
\hline MSE & 533.4604 & 541.2894 & 539.2893 & 469.6580 \\
\hline $\begin{array}{l}\text { SNR } \\
\end{array}$ & 11.0521 & 11.0123 & 11.0186 & 11.5688 \\
\hline RMSE & 23.0967 & 23.2656 & 23.2226 & 21.6715 \\
\hline PSNR & 23.4514 & 23.3881 & 23.4042 & 24.0046 \\
\hline ME3 & 32.0036 & 32.1925 & 32.0573 & 30.6391 \\
\hline ME4 & 39.7137 & 39.8797 & 39.6480 & 38.4851 \\
\hline UQI & 0.4632 & 0.5602 & 0.5596 & 0.5545 \\
\hline SSIM & 0.6251 & 0.6489 & 0.6477 & 0.6679 \\
\hline $\mathrm{AD}$ & 0.0587 & 0.07450 & 0.1698 & 0.1045 \\
\hline SC & 0.8793 & 0.8705 & 0.8741 & 0.8934 \\
\hline NK & 0.9847 & 0.9892 & 0.9871 & 0.9858 \\
\hline MD & 183 & 170 & 178 & 173 \\
\hline LMSE & 36.6506 & 37.8488 & 37.4703 & 32.2299 \\
\hline NAE & 0.3416 & 0.3405 & 0.3408 & 0.3082 \\
\hline
\end{tabular}

Original Image
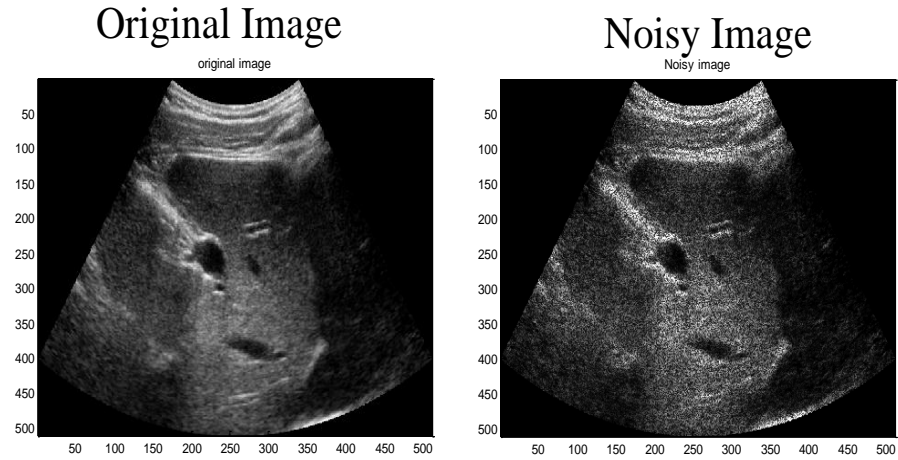
Bilateral Filtering
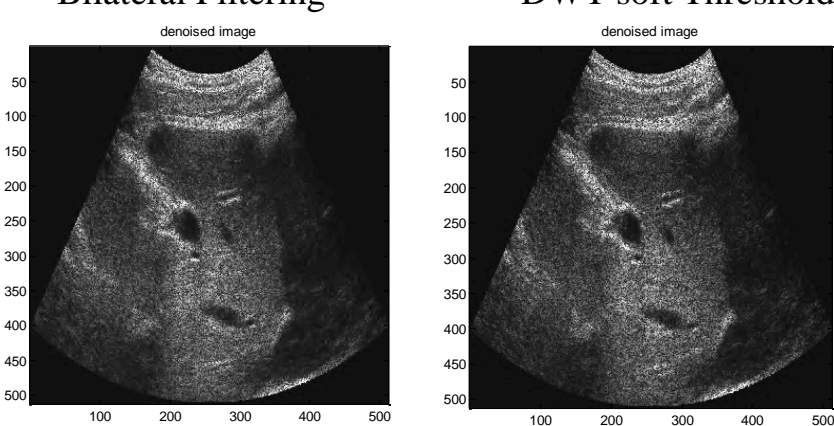

DWT Hard Threshold

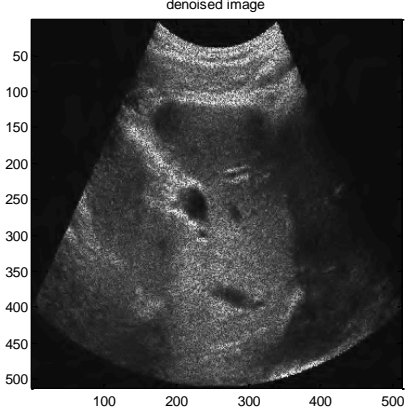

DWT semisoft Threshold

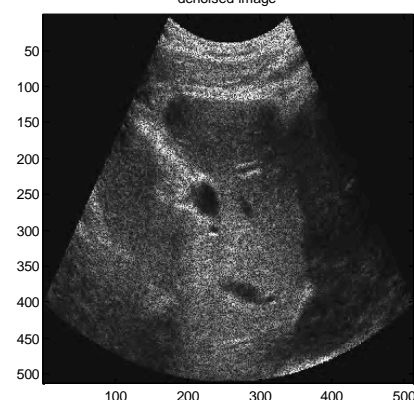

Table 2: Performance evaluation of proposed filter

\begin{tabular}{|c|c|c|c|}
\hline \multirow{2}{*}{$\begin{array}{l}\text { Performance } \\
\text { metrics }\end{array}$} & \multicolumn{3}{|c|}{ Multiresolution Bilateral Filtering } \\
\hline & Soft & Hard & Semisoft \\
\hline MSE & 1.1005 & 0.0269 & 0.0701 \\
\hline SNR & 37.6044 & 53.7333 & 49.5720 \\
\hline RMSE & 1.0490 & 0.1640 & 0.2649 \\
\hline PSNR & 50.3062 & 66.4208 & 62.2598 \\
\hline $\mathrm{ME}$ & 1.2958 & 0.2196 & 0.3482 \\
\hline ME4 & 1.4933 & 0.2713 & 0.4240 \\
\hline UQI & 0.6744 & 0.6846 & 0.6839 \\
\hline SSIM & 0.9941 & 0.9997 & 0.9994 \\
\hline $\mathrm{AD}$ & $3.13 \mathrm{E}-12$ & $3.13 \mathrm{E}-12$ & $3.13 \mathrm{E}-12$ \\
\hline SC & 1.0066 & 1.0000 & 1.0002 \\
\hline NK & 0.9965 & 0.9999 & 0.9998 \\
\hline $\mathrm{MD}$ & 5.9894 & 1.7016 & 2.6631 \\
\hline LMSE & 0.0350 & 0.0026 & 0.0062 \\
\hline $\mathrm{NAE}$ & 0.0191 & 0.0027 & 0.0045 \\
\hline
\end{tabular}

Proposed method with soft Threshold

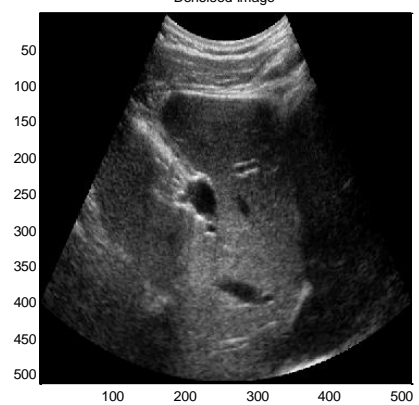

Proposed method with hard Threshold

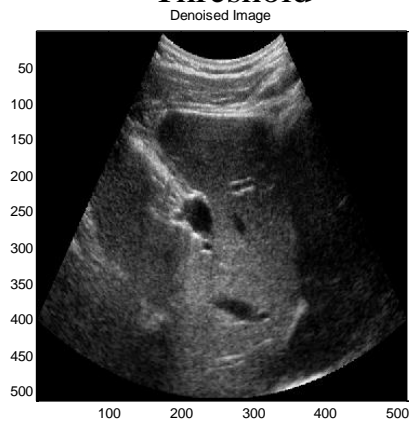

Proposed method with semisoft Threshold

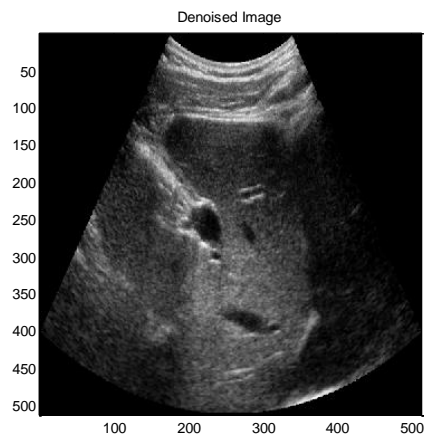

From the above results it was found that the DWT based denoising will remove the noise in detail subbands and the approximation subband is untouched. So the noise in low frequencies is still present after performing the denoising operation. In the proposed method we are eliminating the noise in low pass subband by applying bilateral filtering to it. The performance of this hybrid algorithm is better than the DWT based denoising. The performance of this hybrid approach can be improved using the redundant wavelet transforms such as undecimated wavelet transform, Dual tree complex wavelet transform and double density dual tree complex wavelet transforms for filtering the detail subbands.

\section{REFERENCES}

[1] J.W. Goodman, "Some fundamental properties of speckle," J. Opt. Soc. Am., vol. 66, no. 11, pp. 11451149, 1976.

[2] C.B. Burckhardt, "Speckle in ultrasound B-mode scans," IEEE Trans. Sonics Ultrasonics, vol. SU-25, no. 1, pp. $1-6,1978$.

[3] Z. Tao, H. D. Tagare, and J. D. Beaty. Evaluation of four probability distribution models for speckle in clinical cardiac ultrasound images. IEEE Transactions on Medical Imaging, 25(11):1483\{1491, 2006.

[4] P. C. Tay, S. T. Acton, and J. A. Hossack. A stochastic approach to ultrasound despeckling. In Biomedical Imaging: Nano to Macro, 2006. 3rd IEEE International Symposium on, pages 221\{224, 2006.

[5] J.S. Lee, "Digital image enhancement and noise filtering by using local statistics," IEEE Trans. Pattern Anal. Mach. Intell., PAMI-2, no. 2, pp. 165-168, 1980. 
[6] J.S. Lee, "Speckle analysis and smoothing of synthetic aperture radar images," Comp. Graphics Image Process., vol. 17, pp. 24-32, 1981, doi:10.1016/S0146664X(81)80005-6.

[7] J.S. Lee, "Refined filtering of image noise using local statistics," Comput. Graphics Image Process, vol. 15, pp. 380-389, 1981.

[8] V.S. Frost, J.A. Stiles, K.S. Shanmungan, and J.C. Holtzman, "A model for radar images and its application for adaptive digital filtering of multiplicative noise," IEEE Trans. Pattern Anal. Mach. Intell., vol. 4, no. 2, pp. $157-165,1982$.

[9] D.T. Kuan and A.A. Sawchuk, "Adaptive noise smoothing filter for images with signal dependent noise," IEEE Trans. Pattern Anal. Mach. Intell., vol. PAMI-7, no. 2, pp. 165-177, 1985 .

[10] D.T. Kuan, A.A. Sawchuk, T.C. Strand, and P. Chavel, "Adaptive restoration of images with speckle," IEEE Trans. Acoust., vol. ASSP-35, pp. 373-383, 1987, doi:10.1109/TASSP.1987.1165131.

[11]J. Saniie, T. Wang, and N. Bilgutay, "Analysis of homomorphic processing for ultrasonic grain signal characterization," IEEE Trans. Ultrason. Ferroelectr. Freq. Control, vol. 3, pp. 365-375, 1989, doi:10.1109/58.19177.

[12] A. Pizurica, A. M.Wink, E. Vansteenkiste, W. Philips, and J. Roerdink, "A review of wavelet denoising in mri and ultrasound brain imaging," Curr. Med. Imag. Rev., vol. 2, no. 2, pp. 247-260, 2006.

[13] D.L. Donoho, "Denoising by soft thresholding," IEEE Trans. Inform. Theory, vol. 41, pp. 613-627, 1995.

[14] X. Zong, A. Laine, and E. Geiser, "Speckle reduction and contrast enhancement of echocardiograms via multiscale nonlinear processing," IEEE Trans. Med. Imaging, vol. 17 , no. 4, pp. 532-540, 1998.

[15] X. Hao, S. Gao, and X. Gao, "A novel multiscale nonlinear thresholding method for ultrasonic speckle suppressing," IEEE Trans. Med. Imaging, vol. 18, no. 9, pp. 787-794, 1999.

[16]F.N.S Medeiros, N.D.A. Mascarenhas, R.C.P Marques, and C.M. Laprano, "Edge preserving wavelet speckle filtering," in 5th IEEE Southwest Symposium on Image Analysis and Interpretation, Santa Fe, NM, pp. 281-285, April 7-9, 2002, doi:10.1109/IAI.2002.999933.

[17] C. M. Sehgal, "Quantitative relationship between tissue composition and scattering of ultrasound", J.Acoust. Soc. Am., vol. 94, No.3, pp.1944-1952, Oct.1993.
[18] J. T. M. Verhoeven and J. M. Thijssen, "Improvement of lesion detectability by speckle reduction filtering: A quantitative study", Ultrason. Imag., vol. 15, pp.181-204, 1993.

[19] Paul Butler, "Applied Radiological Imaging for Medical Students", I It Edition, Cambridge University Press, 2007.

[20] Rangaraj M. Rangayyan, "Biomedical Signal Analysis A Case study Approach”, IEEE Press, 2005.

[21] Stephane Mallat, "A Wavelet Tour of signal Processing", Elsevier, 2006.

[22] D L Donoho and M. Jhonstone, "Wavelet shrinkage: Asymptopia? ", J.Roy.Stat.Soc., SerB, Vol.57, pp. 301369, 1995.

[23] D L Donoho, "De-Noising by Soft-Thresholding", IEEE Transactions on Information Theory, vol.41, No.3, May 1995.

[24] David L. Donoho and Iain M. Johnstone.,"Adapting to unknown smoothness via wavelet shrinkage”, Journal of the American Statistical Association, vol.90, no432, pp.1200-1224, December 1995. National Laboratory, July 27, 2001

[25] Ming Zhang and Bahadir K Guntuk, 'Multiresolution bilateral filtering for image denoising', IEEE Transactions on Image Processing, Vol.17, No.12, December 2008.

[26] S. G. Mallat and W. L. Hwang, "Singularity detection and processing with wavelets," IEEE Trans. Inform. Theory, vol. 38, pp. 617-643, Mar. 1992.

[27] I.Daubechies, "Ten Lectures on Wavelets", SIAM Publishers, 1992

[28] Z. Wang, A. C. Bovik, H. R. Sheikh and E. P. Simoncelli, "Image quality assessment: From error visibility to structural similarity," IEEE Transactions on Image Processing, vol. 13, no. 4, pp. 600-612, Apr. 2004.

[29] R. Coifman and D. Donoho, "Translation invariant denoising," in Lecture Notes in Statistics: Wavelets and Statistics, vol. New York: Springer-Verlag, pp. 125--150, 1995.

[32] I.W.Selesnick, "The double density dual tree DWT", IEEE Transactions on Signal Processing, Vol 52. No.5, May 2004. 